QUARTERLY OF APPLIED MATHEMATICS

VOLUME LXVII, NUMBER 4

DECEMBER 2009, PAGES 601-615

S 0033-569X(09)01132-7

Article electronically published on July 29, 2009

\title{
ON A CONTACT PROBLEM IN THERMOELASTICITY WITH SECOND SOUND
}

\author{
$\mathrm{BY}$ \\ JAN SPRENGER \\ Institute for Analysis and Scientific Computing, Vienna University of Technology, Wiedner \\ Hauptstrasse 8-10, 1040 Wien, Austria
}

\begin{abstract}
We investigate the existence and stability of a thermoelastic contact problem with second sound. Previous results established the existence and stability of a solution of the corresponding classical system in the case of radial symmetry. However, recent works have shown that sometimes stability can be lost when the classical Fourier heat conduction is substituted by Cattaneo's Law. We show that also in this case this substitution does indeed lead to a loss in regularity that proves to be a major problem prohibiting the transfer of the existence proof for the classical problem to the problem with second sound, leaving the existence of a solution an open question. We then prove that, if a viscoelastic term is added to the equations, providing additional regularity, then existence and exponential stability (the second, as can be expected, only in the case of radial symmetry) will follow.
\end{abstract}

1. Introduction. We consider a thermoelastic system that can come into contact with a rigid foundation. In particular, consider the equations of thermoelasticity with second sound on a bounded set $\Omega \subset \mathbb{R}^{n}$ with smooth boundary $\partial \Omega=\Gamma_{C} \cup \Gamma_{N} \cup \Gamma_{D}$. On $\Gamma_{D}$, the body is held fix, while on $\Gamma_{N}$ tractions are zero. On $\Gamma_{C}$, the body is free, albeit its extension is limited by a rigid foundation. The temperature is held fixed at the entire boundary. If $u=u(t, x), \theta=\theta(t, x)$ and $q=q(t, x)$ describe the displacement, relative temperature and heat flow, respectively, then our equations take the form

$$
\begin{aligned}
\partial_{t}^{2} u_{i}-\left(C_{i j k l} u_{k, l}\right)_{, j}-\mu \partial_{t} u_{i, j j}+m_{i j} \theta_{, j} & =0 \\
\partial_{t} \theta+\operatorname{div} q+m_{i j} \partial_{t} u_{i, j} & =0 \\
\tau_{0} \partial_{t} q_{i}+q_{i}+K_{i j} \theta_{, j} & =0 .
\end{aligned}
$$

Received March 18, 2008.

2000 Mathematics Subject Classification. Primary 35Q99.

The author would like to thank Professor Dr. Racke for helpful suggestions and the opportunity to work on this topic.

E-mail address: jsprenger@asc.tuwien.ac.at

(C)2009 Brown University Reverts to public domain 28 years from publication 
On $[0, T] \times \Omega$, we define the initial values

$$
u(0, \cdot)=u_{0}, \quad u_{t}(0, \cdot)=u_{1}, \quad \theta(0, \cdot)=\theta_{0}, \quad q(0, \cdot)=q_{0}
$$

satisfying

$$
u_{0} \in\left(H_{\Gamma_{D}}^{1}(\Omega)\right)^{n}, u_{1} \in\left(L^{2}(\Omega)\right)^{n}, \theta_{0} \in L^{2}(\Omega), q_{0} \in\left(L^{2}(\Omega)\right)^{n}
$$

and the boundary conditions

$$
\begin{aligned}
\left.\theta\right|_{\partial \Omega} & =0 \\
\left.u\right|_{\Gamma_{D}} & =0,\left.\quad \sigma_{T}\right|_{\Gamma_{N}}=0 \\
\sigma_{\nu} \leq 0 ; \quad u_{\nu} \leq 0 ; \quad \sigma_{\nu}\left(u_{\nu}-g\right) & =0, \quad \sigma_{T}=0 \text { on } \Gamma_{C}
\end{aligned}
$$

where

$$
\sigma_{i j}=C_{i j k l} u_{k, l}+\mu u_{i, j}-m_{i j} \theta
$$

is the stress tensor and (with $\nu$ being the exterior normal vector),

$$
\sigma_{\nu}=\sigma_{i j} \nu_{i} \nu_{j}, \quad \sigma_{T}=\sigma \nu-\sigma_{\nu} \nu
$$

are its normal and tangential components. We assume the elasticity module $C=$ $\left(C_{i j k l}\right)_{i, j, k, l}$ and that the thermal expansion tensor $m$ and the heat conduction tensor $K$ satisfy

$$
\begin{gathered}
C_{i j k l} \in L^{\infty}(\Omega), \quad \exists d_{C}>0 \forall \eta \in \mathbb{R}^{n \times n}: \eta_{i j} C_{i j k l} \eta_{k l} \geq d_{C}|\eta|^{2}, \quad C_{i j k l}=C_{j i k l}=C_{k l i j}, \\
k_{i j} \in L^{\infty}(\Omega), \quad \exists d_{k}>0 \forall \xi \in \mathbb{R}^{n}: \xi_{i} k_{i j} \xi_{j} \geq d_{k}|\xi|^{2}, \quad k_{i j}=k_{j i}, \\
m_{i j} \in L^{\infty}(\Omega), \quad m_{i j} \geq 0, \quad m_{i j}=m_{j i},
\end{gathered}
$$

where $k=K^{-1}$ in the sense of matrix inversion and $\mu \geq 0$ is (for now) an arbitrary constant.

A few remarks are needed on notation. We denote $\partial_{j} u=u_{, j},\|\cdot\|:=\|\cdot\|_{\left(L^{2}\right)^{m}}$, where $m$ is either $1, n$ or $n^{2}$, which will be clear from the context. In addition, $L^{\infty}\left(H^{1}\right):=L^{\infty}\left([0, T], H^{1}(\Omega)\right)$ and likewise. $H_{\Gamma_{C}}^{1}(\Omega)$ denotes the space of weakly differentiable functions satisfying $\left.u\right|_{\Gamma_{C}}=0$ in a weak sense. The technical problem in the handling of these equations lies in the boundary conditions for $u$ on $\Gamma_{C}$, which do not allow the well-known semi-group theoretic approach. Problems of this form arise naturally in the manufacturing of casts and pistons; cf. [8].

On the classical problem, i.e $\tau_{0}=\mu=0$, there are a number of papers available. In particular, Muñoz Rivera and Racke [6] studied the corresponding classical problem and derived existence and stability under the condition of radial symmetry. In the case of one space dimension, there are several results: Elliott and Tang [2] gave an existence result for more general boundary conditions; Muñoz Rivera and Jiang [5] gave an existence and stability result for a contact problem of two rods, and Gao and Muñoz Rivera [3] gave an existence and stability result for the semilinear case. Dropping the $\partial_{t}^{2} u$ term in the first equation, one arrives at the quasi-statical case, where Shi and Shillor [8] proved the existence of a solution and Ames and Payne [1] gave a uniqueness result. Muñoz Rivera and Racke [6] also prove the existence of a unique solution to the corresponding classical quasi-static problem and its exponential stability. One would (and, in fact, has for quite some time) expect these results to carry over to the fully hyperbolic problem, 
especially since the critical equation for the displacement $u$, where the difficult boundary conditions, arise remains unchanged. However, in a recent work, Racke and Ferñandez Sare [7] showed that for a damped Timoshenko system, exponential stability is lost when substituting the Fourier Law of heat conduction by Cattaneo's Law. In this light, the investigation of the behaviour of this particular system under a transition from classical to hyperbolic heat conduction poses an interesting question. We shall indeed see that this transition leads to a loss in regularity that is not easily compensated, thus requiring the additional viscoelastic term $(\mu>0)$.

This paper is organized as follows: In Section 2, we will give a proof for the existence of a weak solution. We will start by following the approach of Muñoz Rivera and Racke [6] and then show why it cannot be extended to this problem. To this end we will approximate the difficult boundary conditions on $\Gamma_{C}$ and obtain a penalized problem. We will then show that this penalized problem has a solution and give a sufficient condition for the convergence of this solution to a solution of our original problem. This is where the loss of regularity from the changed heat equation leaves its mark, as the conditions derived by Muñoz Rivera and Racke will no longer be sufficient. Finally, in section 3, we will prove a stability result in the radially symmetrical case, that is, the solutions to our problem decay to 0 exponentially. We will use a Lyapunov functional, similar to [6], although some changes are required to compensate for the different heat equation.

2. Existence. We will prove the existence of a solution in the following sense:

Definition 2.1. $(u, \theta, q)$ is a solution to (1.1)-1.7) iff

$$
\begin{gathered}
u \in W^{1, \infty}\left(\left(L^{2}\right)^{n}\right) \cap L^{\infty}\left(\left(H_{\Gamma_{D}}^{1}\right)^{n}\right), \quad \theta \in L^{\infty}\left(L^{2}\right), \quad q \in L^{\infty}\left(\left(L^{2}\right)^{n}\right), \\
\partial_{t} u(T, \cdot), q(T, \cdot) \in\left(L^{2}(\Omega)\right)^{n} ; \quad \theta(T, \cdot) \in L^{2}(\Omega) ; \quad \nabla u(T, \cdot) \in L^{2}(\Omega), \\
\forall w \in W^{1, \infty}\left(\left(L^{2}\right)^{n}\right) \cap L^{\infty}\left(\left(H_{\Gamma_{D}}^{1}\right)^{n}\right), w_{\nu} \leq g \text { on } \Gamma_{C}: \\
-\int_{0}^{T}\left\langle\partial_{t} u, \partial_{t} w\right\rangle \mathrm{d} t+\left\langle u(T, \cdot), \partial_{t} w(T, \cdot)-\partial_{t} u(T, \cdot)\right\rangle-\left\langle u_{0}, \partial_{t} w(0, \cdot)-u_{1}\right\rangle \\
+\mu \int_{0}^{T}\left\langle\partial_{t} u_{i, j}, w_{i, j}\right\rangle \mathrm{d} t+\int_{0}^{T}\left\langle C_{i j k l} u_{k, l}, w_{i, j}\right\rangle \mathrm{d} t-\int_{0}^{T}\left\langle m_{i j} \theta, w_{i, j}\right\rangle \mathrm{d} t \\
+\int_{0}^{T}\left\langle\partial_{t} u, \partial_{t} u\right\rangle \mathrm{d} t-\int_{0}^{T}\left\langle C_{i j k l} u_{k, l}, u_{i, j}\right\rangle \mathrm{d} t-\frac{\mu}{2}\left(\|\nabla u(T, \cdot)\|^{2}-\left\|\nabla u_{0}\right\|^{2}\right) \\
+\int_{0}^{T}\left\langle m_{i j} \theta, u_{i j}\right\rangle \mathrm{d} t \geq 0 ; \\
\forall z \in W^{1, \infty}\left(H_{0}^{1}\right): \\
-\int_{0}^{T}\langle\theta, z\rangle \mathrm{d} t+\langle\theta(T, \cdot), z(T, \cdot)\rangle-\left\langle\theta_{0}, z(0, \cdot)\right\rangle-\int_{0}^{T}\left\langle q_{i}, z, i\right\rangle \mathrm{d} t \\
-\int_{0}^{T}\left\langle m_{i j} u_{i, j}, \partial_{t} z\right\rangle \mathrm{d} t+\left\langle m_{i j} u_{i, j}(T, \cdot), z(T, \cdot)\right\rangle-\left\langle m_{i j} u_{0 i, j}, z(0, \cdot)\right\rangle=0 ; \\
\forall y \in W^{1, \infty}\left(\left(H^{1}\right)^{n}\right):
\end{gathered}
$$




$$
\begin{gathered}
-\tau_{0} \int_{0}^{T}\left\langle k_{i j} q_{i}, \partial_{t} y_{j}\right\rangle \mathrm{d} t+\tau_{0}\left\langle k_{i j} q_{i}(T, \cdot), y_{j}(T, \cdot)\right\rangle-\tau_{0}\left\langle k_{i j} q_{0 i}, y_{j}(0, \cdot)\right\rangle \\
+\int_{0}^{T}\left\langle k_{i j} q_{i}, y_{j}\right\rangle \mathrm{d} t-\int_{0}^{T}\left\langle\theta, y_{i, i}\right\rangle \mathrm{d} t=0 \\
\left.u\right|_{\Gamma_{C}} \leq g \text { a.e. }
\end{gathered}
$$

We remark that all boundary conditions are represented in a weak sense in the above definiton. Also, we need the unusual condition (2.2) for condition (2.3) to make sense. This will be seen from the context in section 2 .

To better handle the difficult boundary conditions in $u$, we consider the following penalized problem:

$$
\begin{aligned}
\partial_{t}^{2} u_{i}^{\epsilon}-\left(C_{i j k l} u_{k, l}^{\epsilon}\right)_{, j}-\mu u_{i, j j}^{\epsilon}+m_{i j} \theta_{, j}^{\epsilon}=0 \\
\partial_{t} \theta^{\epsilon}+\operatorname{div} q^{\epsilon}+m_{i j} \partial_{t} u_{i, j}^{\epsilon}=0 \\
\tau_{0} \partial_{t} k_{i j} q_{j}^{\epsilon}+k_{i j} q_{j}^{\epsilon}+\theta_{, i}^{\epsilon}=0,
\end{aligned}
$$

with initial conditions

$$
u(0, \cdot)=u_{0}, \quad u_{t}(0, \cdot)=u_{1}, \quad \theta(0, \cdot)=\theta_{0}, \quad q(0, \cdot)=q_{0}
$$

and boundary conditions

$$
\begin{aligned}
\left.\theta\right|_{\partial} \Omega=0, & \\
\left.u\right|_{\Gamma_{D}}=0, & \left.\sigma_{T}\right|_{\Gamma_{N}}=0, \\
\sigma_{\nu}^{\epsilon}=-\frac{1}{\epsilon}\left(u_{\nu}^{\epsilon}-g\right)^{+}-\epsilon \partial_{t} u_{\nu}, & \sigma_{T}=0 \text { on } \Gamma_{C} .
\end{aligned}
$$

Note that only the boundary conditions on $\Gamma_{C}$ have been changed; everything else is identical to the original problem. We will see that $\sigma_{\nu}^{\epsilon}$ is bounded and therefore by (2.11), $\left(u_{\nu}^{\epsilon}-g\right)^{+} \rightarrow 0$ as $\epsilon \rightarrow 0$, satisfying (2.6). Next, we give a definition of a solution to the penalized problem. Let $w^{p}{ }_{p}, y^{p}{ }_{p} \subset H^{1}(\Omega)$ be bases of $\left(L^{2}(\Omega)\right)^{n}$ and $z^{p}{ }_{p} \subset H_{0}^{1}(\Omega)$ be a basis of $L^{2}(\Omega)$.

DeFinition 2.2. Let

$$
\begin{aligned}
u_{0}^{\varepsilon}, u_{1}^{\varepsilon} & \in\left(H^{2,2}(\Omega) \cap H_{0}^{1}(\Omega)\right)^{n}, \\
q_{0}^{\varepsilon} & \in\left(H^{1}(\Omega)\right)^{n} \\
\theta_{0}^{\epsilon} & \in H_{0}^{1}(\Omega) .
\end{aligned}
$$

Then $\left(u^{\epsilon}, \theta^{\epsilon}, q^{\epsilon}\right)$ is a solution to (2.7)-(2.11) iff

$$
\begin{gathered}
u^{\varepsilon} \in W^{2, \infty}\left(\left(L^{2}\right)^{n}\right) \cap W^{1, \infty}\left(\left(H_{\Gamma_{D}}^{1}\right)^{n}\right) ; \quad \theta^{\varepsilon} \in W^{1, \infty}\left(L^{2}\right) \cap L^{\infty}\left(H_{0}^{1}\right) ; \\
q^{\varepsilon} \in W^{1, \infty}\left(\left(L^{2}\right)^{n}\right) \cap L^{\infty}\left(D^{1}\right) ; \\
u^{\varepsilon}(0, \cdot)=u_{0}^{\varepsilon} ; \quad \partial_{t} u^{\varepsilon}(0, \cdot)=u_{1}^{\varepsilon} ; \quad \theta^{\varepsilon}(0, \cdot)=\theta_{0}^{\varepsilon} ; \quad q^{\varepsilon}(0, \cdot)=q_{0}^{\varepsilon}
\end{gathered}
$$


and for almost all $t \in[0, T]$,

$$
\begin{gathered}
\forall p \in \mathbb{N}:\left\langle\partial_{t}^{2} u^{\varepsilon}(t, \cdot), w^{p}\right\rangle+\mu\left\langle\partial_{t} u_{i, j}^{\varepsilon}(t, \cdot), w_{i, j}^{p}\right\rangle+\left\langle C_{i j k l} u_{k, l}^{\varepsilon}(t, \cdot), w_{i, j}^{p}\right\rangle \\
-\left\langle m_{i j} \theta^{\varepsilon}(t, \cdot), w_{i, j}^{p}\right\rangle=-\frac{1}{\varepsilon} \int_{\Gamma_{C}}\left(u_{\nu}^{\varepsilon}(t, \cdot)-g\right)^{+} w^{p} \mathrm{~d} \Gamma-\varepsilon \int_{\Gamma_{C}} \partial_{t} u_{\nu}^{\varepsilon}(t, \cdot) w^{p} \mathrm{~d} \Gamma, \\
\forall p \in \mathbb{N}:\left\langle\partial_{t} \theta^{\varepsilon}(t, \cdot), z^{p}\right\rangle+\left\langle\operatorname{div} q^{\varepsilon}(t, \cdot), z^{p}\right\rangle+\left\langle m_{i j} \partial_{t} u_{i, j}^{\varepsilon}(t, \cdot), z^{p}\right\rangle=0, \\
\forall p \in \mathbb{N}: \tau_{0}\left\langle k_{i j} \partial_{t} q_{i}^{\varepsilon}(t, \cdot), y_{j}^{p}\right\rangle+\left\langle k_{i j} q_{i}^{\varepsilon}(t, \cdot), y_{j}^{p}\right\rangle+\left\langle\nabla \theta^{\varepsilon}(t, \cdot), y_{i}^{p}\right\rangle=0 .
\end{gathered}
$$

To construct a solution to the penalized problem, we will use a Faedo-Galerkin method. Note that, if $(v, \psi, h)$ satisfy

$$
\begin{gathered}
v(0, \cdot)=\partial_{t} v(0, \cdot)=\psi(0, \cdot)=h(0, \cdot)=0, \\
\left\langle\partial_{t}^{2} v, w^{p}\right\rangle+\left\langle\left(C_{i j k l} v_{k, l}\right), w_{i, j}^{p}\right\rangle+\mu\left\langle\partial_{t} v_{i, j}, w_{i, j}^{p}\right\rangle-\left\langle m_{i j} \psi, w_{i, j}^{p}\right\rangle \\
=\left\langle f, w^{p}\right\rangle-\frac{1}{\varepsilon} \int_{\Gamma_{C}}\left(v_{\nu}-g\right)^{+} w_{\nu}^{p} \mathrm{~d} \Gamma-\varepsilon \int_{\Gamma_{C}}\left(\partial_{t} v_{\nu}\right) w_{\nu}^{p} \mathrm{~d} \Gamma, \\
\left\langle\partial_{t} \psi, z^{p}\right\rangle+\left\langle\operatorname{div} h, z^{p}\right\rangle+\left\langle m_{i j} \partial_{t} v_{i, j}, z^{p}\right\rangle=\left\langle b, z^{p}\right\rangle, \\
\left\langle\tau_{0} k_{i j} \partial_{t} h_{j}, y_{i}\right\rangle+\left\langle k_{i j} h_{j}, y_{i}\right\rangle+\langle\nabla \psi, y\rangle=\left\langle e, y^{p}\right\rangle,
\end{gathered}
$$

with

$$
\begin{aligned}
f_{i} & \left.:=C_{i j k l}\left(u_{0 k, l}^{\varepsilon}-t u_{1 k, l}^{\varepsilon}\right)\right)_{, j}+\mu u_{1 i, j j}^{\varepsilon}-\left(m_{i j} \theta_{0}^{\varepsilon}\right)_{, j} \quad(i=1, \ldots, n) \\
b & :=-q_{0 i, i}^{\varepsilon}+m_{i j} u_{1 i, j}^{\varepsilon} \\
e & :=-k_{i j} q_{0 j}^{\varepsilon}-\theta_{0, j}^{\varepsilon}
\end{aligned}
$$

then $u:=v+u_{0}+t u_{1}, \theta:=\psi+\theta_{0}$ and $q:=h+q_{0}$ are a solution to the penalized problem. To find such $(v, \psi, h)$, consider the following set of equations on $[0, T]$ :

$$
\begin{gathered}
\left\langle\partial_{t}^{2} v^{m}, w^{p}\right\rangle_{n}+\left\langle C_{i j k l} v_{k, l}^{m}, w_{i, j}^{p}\right\rangle+\mu\left\langle\partial_{t} v_{i, j}^{m}, w_{i, j}^{p}\right\rangle-\left\langle m_{i j} \psi, w_{i, j}^{p}\right\rangle \\
=\left\langle f, w^{p}\right\rangle_{n}-\frac{1}{\varepsilon} \int_{\Gamma_{C}}\left(v_{\nu}^{m}-g\right)^{+} w_{\nu}^{p} \mathrm{~d} \Gamma-\varepsilon \int_{\Gamma_{C}} \partial_{t} v_{\nu}^{m} w_{\nu}^{p} \mathrm{~d} \Gamma \quad(p=1, \ldots, m), \\
\left\langle\partial_{t} \psi^{m}, z^{p}\right\rangle+\left\langle\operatorname{div} h^{m}, z^{p}\right\rangle+\left\langle m_{i j} \partial_{t} v_{i, j}^{m}, z^{p}\right\rangle=\left\langle b, z^{p}\right\rangle(p=1, \ldots, m), \\
\tau_{0}\left\langle k_{i j} \partial_{t} h_{i}^{m}, y_{j}^{p}\right\rangle+\left\langle k_{i j} h_{i}^{m}, y_{j}^{p}\right\rangle+\left\langle\nabla \psi^{m}, y^{p}\right\rangle_{n}=\left\langle e, y^{p}\right\rangle_{n}(p=1, \ldots, m), \\
v(0, \cdot)=\partial_{t} v(0, \cdot)=\psi(0, \cdot)=h(0, \cdot)=0,
\end{gathered}
$$

where $v^{m}(t, x)=a_{p}^{m}(t) w^{p}(x), \psi^{m}(t, x)=b_{p}^{m}(t) z^{p}(x)$ and $h^{m}(t, x)=c_{p}^{m}(t) y^{p}(x)$ with unknown coefficents $\left(a_{p}^{m}, b_{p}^{m}, c_{p}^{m}\right)$. Then (2.20)-(2.23) is a set of ordinary differential equations for $\left(a_{p}^{m}, b_{p}^{m}, c_{p}^{m}\right)$, thus possessing a solution with the regularity

$$
v^{m} \in W^{3, \infty}\left(\left(H_{\Gamma_{D}}^{1}\right)^{n}\right), \psi^{m} \in W^{2, \infty}\left(H_{0}^{1}\right), h^{m} \in W^{2, \infty}\left(\left(H^{1}\right)^{n}\right) .
$$

Note that the initial conditions are arbitrarily smooth and that $f, g, e$ are polynomial in $t$, allowing for a solution with the required smoothness. 
LEMma 2.3. There exist $(v, \psi, h)$ such that

$$
\begin{aligned}
\left(v^{m}\right)_{m} & \stackrel{*}{*} v \text { in } W^{2, \infty}\left(\left(L^{2}\right)^{n}\right) \cap W^{1, \infty}\left(\left(H_{\Gamma_{C}}^{1}\right)^{n}\right), \\
\left(\psi^{m}\right)_{m} & \stackrel{*}{\rightarrow} \psi \text { in } W^{1, \infty}\left(L^{2}\right), \\
\left(h^{m}\right)_{m} & \stackrel{*}{*} h \text { in } W^{1, \infty}\left(\left(L^{2}\right)^{n}\right) .
\end{aligned}
$$

Proof. Multiplying (2.20) by $\frac{\mathrm{d}}{\mathrm{d} t} a_{p}^{m}$, (2.21) by $b_{p}^{m}$ and (2.22) by $c_{p}^{m}$, respectively, we obtain after summarizing from 1 to $m$ :

$$
\begin{aligned}
& \frac{1}{2} \frac{\mathrm{d}}{\mathrm{d} t}\left(\left\|\partial_{t} v^{m}\right\|^{2}+\left\langle C_{i j k l} v_{k, l}^{m}, v_{i, j}^{m}\right\rangle+\frac{1}{\varepsilon} \int_{\Gamma_{C}}\left|\left(u_{\nu}^{m}-g\right)^{+}\right|^{2} \mathrm{~d} \Gamma+\left\|\psi^{m}\right\|^{2}+\tau_{0}\left\|h^{m}\right\|^{2}\right) \\
& +\mu\left\|\partial_{t} \nabla v^{m}\right\|^{2}+\varepsilon \int_{\Gamma_{C}}\left|\partial_{t} v_{\nu}^{m}\right|^{2} \mathrm{~d} \Gamma+\left\langle k_{i j} h_{i}^{m}, h_{j}^{m}\right\rangle+\left\langle\operatorname{div} h^{m}, \psi^{m}\right\rangle+\left\langle\nabla \psi^{m}, h^{m}\right\rangle \\
= & \left\langle f, \partial_{t} v^{m}\right\rangle+\left\langle b, \psi^{m}\right\rangle+\left\langle e, h^{m}\right\rangle,
\end{aligned}
$$

where we used that

$$
\int_{\Gamma_{C}}\left(v_{\nu}^{m}-g\right)^{+} \partial_{t} v^{m} \mathrm{~d} \Gamma=\frac{\mathrm{d}}{\mathrm{d} t} \int_{\Gamma_{C}}\left|\left(v_{\nu}^{m}-g\right)^{+}\right|^{2} \mathrm{~d} \Gamma .
$$

As one easily checks by partial integration,

$$
\left\langle\operatorname{div} h^{m}, \psi^{m}\right\rangle+\left\langle\nabla \psi^{m}, h^{m}\right\rangle=0
$$

and therefore, after integrating (2.24) on $(0, t)$, we obtain by Gronwall's inequality,

$$
\begin{aligned}
\left\|\partial_{t} v^{m}(t, \cdot)\right\|_{n} & \leq C, \\
\left\langle C_{i j k l} v_{k, l}^{m}(t, \cdot), v_{i, j}^{m}(t, \cdot)\right\rangle & \leq C, \\
\frac{1}{\varepsilon} \int_{\Gamma_{C}}\left|\left(v_{\nu}^{m}(t, \cdot)-g\right)^{+}\right|^{2} \mathrm{~d} \Gamma & \leq C, \\
\left\|h^{m}(t, \cdot)\right\|_{n} & \leq C, \\
\varepsilon \int_{0}^{t} \int_{\Gamma_{C}}\left|\partial_{t} v_{\nu}^{m}(t, \cdot)\right|^{2} \mathrm{~d} \Gamma \mathrm{d} t & \leq C, \\
\int_{0}^{t}\left\langle k_{i j} h_{i}^{m}(t, \cdot), h_{j}^{m}(t, \cdot)\right\rangle \mathrm{d} t & \leq C .
\end{aligned}
$$

Using the smoothness of the functions $\left(v^{m}, \psi^{m}, h^{m}\right)$, we see that they satisfy the timederivated system

$$
\begin{gathered}
\left\langle\partial_{t}^{3} v^{m}, w^{p}\right\rangle_{n}+\left\langle C_{i j k l} \partial_{t} v_{k, l}^{m}, w_{i, j}^{p}\right\rangle+\mu\left\langle\partial_{t}^{2} v_{i, j}^{m}, w_{i, j}^{p}\right\rangle-\left\langle m_{i j} \partial_{t} \psi, w_{i, j}^{p}\right\rangle \\
=-\frac{1}{\varepsilon} \int_{\Gamma_{C}} \partial_{t}\left(v_{\nu}^{m}-g\right)^{+} w_{\nu}^{p} \mathrm{~d} \Gamma-\varepsilon \int_{\Gamma_{C}} \partial_{t}^{2} v_{\nu}^{m} w_{\nu}^{p} \mathrm{~d} \Gamma+\left\langle\partial_{t} f, w^{p}\right\rangle_{n}, \\
\left\langle\partial_{t}^{2} \psi^{m}, z^{p}\right\rangle+\left\langle\partial_{t} \operatorname{div} h^{m}, z^{p}\right\rangle+\left\langle m_{i j} \partial_{t}^{2} v_{i, j}^{m}, z^{p}\right\rangle=0 \\
\tau_{0}\left\langle k_{i j} \partial_{t}^{2} h_{i}^{m}, y_{j}^{p}\right\rangle+\left\langle k_{i j} \partial_{t} h_{i}^{m}, y_{j}^{p}\right\rangle+\left\langle\partial_{t} \nabla \psi^{m}, y^{p}\right\rangle_{n}=0 .
\end{gathered}
$$


Multiplying (2.27) by $\frac{\mathrm{d}^{2}}{\mathrm{~d} t^{2}} a_{p}^{m},(2.28)$ by $\frac{\mathrm{d}}{\mathrm{d} t} b_{p}^{m}$ and (2.29) by $\frac{\mathrm{d}}{\mathrm{d} t} c_{p}^{m}$, respectively, we obtain similar to (2.24),

$$
\begin{aligned}
& \frac{1}{2} \frac{\mathrm{d}}{\mathrm{d} t}\left[\left\|\partial_{t}^{2} v^{m}\right\|_{n}^{2}+\left\langle C_{i j k l} \partial_{t} v_{k, l}^{m}, \partial_{t} v_{i, j}^{m}\right\rangle+\left\|\partial_{t} \psi^{m}\right\|^{2}+\tau_{0}\left\|\partial_{t} h^{m}\right\|_{n}^{2}\right] \\
+ & \mu\left\|\partial_{t}^{2} \nabla v^{m}\right\|_{n \times n}^{2}+\left\langle k_{i j} h_{i}^{m}, h_{j}^{m}\right\rangle \\
= & \left\langle\partial_{t} f, \partial_{t}^{2} v^{m}\right\rangle-\frac{1}{\varepsilon} \int_{\Gamma_{C}} \partial_{t}\left(v_{\nu}^{m}-g\right)^{+} \partial_{t}^{2} v^{m} \mathrm{~d} \Gamma-\varepsilon \int_{\Gamma_{C}} \partial_{t}^{2} v_{\nu}^{m} \partial_{t}^{2} v_{\nu}^{m} \mathrm{~d} \Gamma .
\end{aligned}
$$

Observe that in general it is not

$$
\partial_{t}\left|\partial_{t}\left(v_{\nu}^{m}-g\right)^{+}\right|^{2}=2 \partial_{t}\left(v_{\nu}^{m}-g\right)^{+} \partial_{t}^{2} v^{m} \text { a.e. }
$$

since the distributional second derivative of $\left(v_{\nu}^{m}-g\right)^{+}$need not be regular. However, using (2.25),

$$
\begin{aligned}
& \frac{1}{2} \frac{\mathrm{d}}{\mathrm{d} t}\left[\left\|\partial_{t}^{2} v^{m}\right\|_{n}^{2}+\left\langle C_{i j k l} \partial_{t} v_{k, l}^{m}, \partial_{t} v_{i, j}^{m}\right\rangle+\left\|\partial_{t} \psi^{m}\right\|^{2}+\tau_{0}\left\|\partial_{t} h^{m}\right\|_{n}^{2}\right] \\
& +\mu\left\|\partial_{t}^{2} \nabla v^{m}\right\|_{n \times n}^{2}+\left\langle k_{i j} h_{i}^{m}, h_{j}^{m}\right\rangle \\
\leq & \left\langle\partial_{t} f, \partial_{t}^{2} v^{m}\right\rangle_{n}+\frac{1}{2 \varepsilon^{3}} \int_{\Gamma_{C}}\left|\partial_{t} v_{\nu}^{m}\right|^{2} \mathrm{~d} \Gamma-\frac{\varepsilon}{2} \int_{\Gamma_{C}}\left|\partial_{t}^{2} v_{\nu}^{m}\right|^{2} \mathrm{~d} \Gamma \\
\leq & \left\langle\partial_{t} f, \partial_{t}^{2} v^{m}\right\rangle_{n}+C_{\varepsilon},
\end{aligned}
$$

where $C^{\epsilon} \rightarrow \infty$ as $\epsilon \rightarrow 0$. For constant $\epsilon$ we conclude, using Gronwall's inequality again, that

$$
\begin{array}{lll}
\left(v^{m}\right)_{m} & \text { is bounded in } & W^{2, \infty}\left(\left(L^{2}\right)^{n}\right) \cap W^{1, \infty}\left(\left(H_{\Gamma_{D}}^{1}\right)^{n}\right), \\
\left(\psi^{m}\right)_{m} & \text { is bounded in } & W^{1, \infty}\left(L^{2}\right), \\
\left(h^{m}\right)_{m} & \text { is bounded in } & W^{1, \infty}\left(\left(L^{2}\right)^{n}\right),
\end{array}
$$

from which the claimed convergence follows.

We can now show

Theorem 2.4. There is a solution to the penalized problem.

Proof. Take $(v, \psi, h)$ as in Lemma 2.3. Define

$$
\begin{gathered}
u:=v+u_{0}+t u_{1}, \\
\theta:=\psi+\theta_{0}, \\
q:=h+q_{0} .
\end{gathered}
$$

Then it is clear that $(u, \theta, q)$ have the desired regularity (2.12) and fulfill the initial conditions (2.13). Using Lemma 1.4 from [4, we obtain the convergence

$$
u^{\varepsilon} \rightarrow u \text { in } C^{1}\left([0, T],\left(L^{2}\left(\Gamma_{C}\right)\right)^{n}\right) .
$$

It then follows from the convergence proved in Theorem 2.3 that $(u, \theta, q)$ satisfy (2.14)(2.16).

Now we will prove the convergence of solutions to the penalized problem. As we can see in the proof of Lemma (2.3), we cannot use the second energy level to gain estimates on the convergence of $\left(u^{\epsilon}, \theta^{\epsilon}, q^{\epsilon}\right)$, as $\epsilon$ is now no longer constant. Therefore, we lose one 
level of regularity in time. This loss is grave, since we will no longer have convergence of some terms in the equations; i.e., it is generally unknown if the limits $(u, \psi, q)$ are solutions to the original problem. However, if $\mu>0$, the viscoelastic term will provide us with the missing regularity and an existence proof is possible. This will be shown in detail in the proof of Theorem 2.7

Lemma 2.5. There exist $(u, \theta, q)$ such that

$$
\begin{array}{lll}
u^{\varepsilon} \stackrel{*}{\rightarrow} u & \text { in } & W^{1, \infty}\left(\left(L^{2}\right)^{n}\right) \cap L^{\infty}\left(H_{\Gamma_{C}}^{1}\right), \\
\theta^{\varepsilon} \stackrel{*}{\rightarrow} \theta & \text { in } & L^{\infty}\left(L^{2}\right), \\
q^{\varepsilon} \stackrel{*}{*} q & \text { in } & L^{\infty}\left(\left(L^{2}\right)^{n}\right) .
\end{array}
$$

If $\mu>0$, then

$$
u^{\varepsilon} \rightarrow u \text { in } W^{1,2}\left(\left(H_{\Gamma_{C}}^{1}\right)^{n}\right) .
$$

Proof. By the regularity of $\left(u^{\varepsilon}, \theta^{\epsilon}, q^{\epsilon}\right)$, we can subsitute them for $\left(w^{p}, z^{p}, y^{p}\right)$ in (2.14), (2.15) and (2.16) respectively and obtain

$$
\begin{aligned}
& \frac{\mathrm{d}}{\mathrm{d} t}\left(\left\|\partial_{t} u^{\varepsilon}\right\|^{2}+\left\langle C_{i j k l} u_{k, l}^{\varepsilon}, u_{i, j}^{\varepsilon}\right\rangle+\left\|\theta^{\varepsilon}\right\|^{2}+\left\langle k_{i j} q_{i}^{\varepsilon}, q_{j}^{\varepsilon}\right\rangle+\frac{1}{\varepsilon} \int_{\Gamma_{C}}\left|\left(u_{\nu}^{\varepsilon}-g\right)^{+}\right|^{2} \mathrm{~d} \Gamma\right) \\
& +\mu\left\|\partial_{t} \nabla u^{\varepsilon}\right\|^{2}+\left\langle k_{i j} q_{i}^{\varepsilon}, q_{j}^{\varepsilon}\right\rangle+\varepsilon \int_{\Gamma_{C}}\left|\partial_{t} u_{\nu}^{\varepsilon}\right|^{2} \mathrm{~d} \Gamma=0,
\end{aligned}
$$

where we again used that

$$
\langle\operatorname{div} q, \theta\rangle+\langle\nabla \theta, q\rangle=0 .
$$

Integrating from 0 to $t$ and using Gronwall's inequality, we conclude the existence of a constant $C=C\left(\left\|u_{0}^{\varepsilon}\right\|,\left\|\theta_{0}^{\varepsilon}\right\|,\left\|q_{0}^{\varepsilon}\right\|\right)$ such that for all $t>0$,

$$
\begin{aligned}
\left\|\partial_{t} u^{\varepsilon}(t, \cdot)\right\|_{n} & \leq C, \\
\left\langle C_{i j k l} u_{k, l}^{\varepsilon}(t, \cdot), u_{i, j}^{\varepsilon}(t, \cdot)\right\rangle & \leq C, \\
\left\|\theta^{\varepsilon}(t, \cdot)\right\| & \leq C, \\
\left\langle k_{i j} q_{i}^{\varepsilon}(t, \cdot), q_{j}^{\varepsilon}(t, \cdot)\right\rangle & \leq C, \\
\frac{1}{\varepsilon} \int_{\Gamma_{C}}\left|\left(u_{\nu}^{\varepsilon}(t, \cdot)-g(\cdot)\right)^{+}\right|^{2} \mathrm{~d} \Gamma & \leq C, \\
\mu \int_{0}^{t}|| \partial_{t} \nabla u^{\varepsilon}(s, \cdot) \|_{n}^{2} \mathrm{~d} s & \leq C, \\
\int_{0}^{t}\left\langle k_{i j} q_{i}^{\varepsilon}(s, \cdot), q_{j}^{\varepsilon}(s, \cdot)\right\rangle \mathrm{d} s & \leq C, \\
\varepsilon \int_{0}^{t} \int_{\Gamma_{C}}\left|\partial_{t} u_{\nu}^{\varepsilon}(s, \cdot)\right|^{2} \mathrm{~d} \Gamma \mathrm{d} s & \leq C .
\end{aligned}
$$

This implies the desired convergence. 
Lemma 2.6. Let $(u, \theta, q)$ be the functions from Lemma 2.5. Then

$$
\begin{array}{rll}
u^{\varepsilon}(T, \cdot) \stackrel{*}{\rightarrow} u(T, \cdot) & \text { in } & L^{\infty}\left(H_{\Gamma_{C}}^{1}\right), \\
u_{t}^{\varepsilon}(T, \cdot) \stackrel{*}{*} u_{t}(T, \cdot) & \text { in } & L^{\infty}\left(L^{2}\right), \\
\theta^{\varepsilon}(T, \cdot) \stackrel{*}{\rightarrow} \theta(T, \cdot) & \text { in } & L^{\infty}\left(L^{2}\right), \\
q^{\varepsilon}(T, \cdot) \stackrel{*}{*} q(T, \cdot) & \text { in } & L^{\infty}\left(L^{2}\right) .
\end{array}
$$

Proof. Note that due to the regularity of the solutions to the penalized problem, $u^{\epsilon}, u_{t}^{\epsilon}, \theta^{\epsilon}$ and $q^{\epsilon}$ are continuous in time by Sobolev's Imbedding Theorem. Therefore, the asserted convergence holds by the estimates gained in the proof for Theorem 2.5.

TheOrem 2.7. Let $\mu>0$. Let $\left(u_{0}, u_{1}, \theta_{0}, q_{0}\right) \in\left(H_{\Gamma_{D}}^{1}(\Omega)\right)^{n} \times\left(L^{2}(\Omega)\right)^{2 n+1}$. Then there exists a solution to (1.1)-1.7.

Proof. Let

$$
\begin{aligned}
\left(u_{0}^{\varepsilon}\right)_{\varepsilon}, & \left(u_{1}^{\varepsilon}\right)_{\varepsilon} \\
\left(q_{0}^{\varepsilon}\right)_{\varepsilon} & \subset\left(H_{0}^{1}(\Omega) \cap H^{2,2}(\Omega)\right)^{n} \\
\left(\theta_{0}^{\varepsilon}\right)_{\varepsilon} & \subset H^{1}(\Omega)
\end{aligned}
$$

with

$$
\begin{aligned}
u_{0}^{\varepsilon} \longrightarrow u_{0} \quad \text { in } & \left(H_{\Gamma_{D}}^{1}\right)^{n}, \\
u_{1}^{\varepsilon} \longrightarrow u_{1} & \text { in } \quad\left(L^{2}(\Omega)\right)^{n}, \\
\theta_{0}^{\varepsilon} \longrightarrow \theta_{0} & \text { in } \quad L^{2}(\Omega), \\
q_{0}^{\varepsilon} \longrightarrow q_{0} & \text { in } \quad\left(L^{2}(\Omega)\right)^{n} .
\end{aligned}
$$

Let $\left(u^{\epsilon}, \theta^{\epsilon}, q^{\epsilon}\right)$ be the solutions to the penalized problem for each $\epsilon>0$ and $(u, \theta, q)$ be the limits from Lemma 2.5. Then $(u, \theta, q)$ will satisfy (2.1). We can substitute $z^{p}$ in (2.15) for any $z \in W^{1, \infty}\left(H_{0}^{1}\right)$ and obtain

$$
\left\langle\partial_{t} \theta^{\varepsilon}, z\right\rangle+\left\langle\operatorname{div} q^{\varepsilon}, z\right\rangle+\left\langle m_{i j} \partial_{t} u_{i, j}^{\varepsilon}, z\right\rangle=0 .
$$

Integrating from 0 to $T$ we arrive at

$$
\begin{gathered}
\left\langle\theta(T, \cdot)^{\varepsilon}, z(T, \cdot)\right\rangle-\left\langle\theta_{0}^{\varepsilon}, z(0, \cdot\rangle-\int_{0}^{T}\left\langle\theta^{\varepsilon}, \partial_{t} z\right\rangle \mathrm{d} t-\int_{0}^{T}\left\langle q_{i}^{\varepsilon}, z_{, i}\right\rangle \mathrm{d} t\right. \\
+\left\langle m_{i j} u_{i, j}^{\varepsilon}(T, \cdot), z(T, \cdot)\right\rangle-\left\langle m_{i j} u_{0 i, j}^{\varepsilon}, z(0, \cdot)\right\rangle-\int_{0}^{T}\left\langle m_{i j} u_{i, j}^{\varepsilon}, z\right\rangle \mathrm{d} t=0 .
\end{gathered}
$$

Using Lemmas 2.5 and 2.6, we conclude, by taking the limit $\epsilon \rightarrow 0$, that $(u, \theta, q)$ fulfill (2.4).

Similarly, substituting $y^{p}$ in (2.16) for any $y \in W^{1, \infty}\left(H^{1}\right)$ and integrating yields

$$
\begin{aligned}
& \left\langle k_{i j} q_{i}^{\varepsilon}(T, \cdot), y(T, \cdot)\right\rangle-\left\langle k_{i j} q_{0} i^{\varepsilon}, y(0, \cdot)\right\rangle-\int_{0}^{T}\left\langle k_{i j} q_{i}^{\varepsilon}(t, \cdot), \partial_{t} y_{j}(t, \cdot)\right\rangle \mathrm{d} t \\
+ & \int_{0}^{T}\left\langle k_{i j} q_{i}^{\varepsilon}(t, \cdot), y_{j}(t, \cdot)\right\rangle+\int_{0}^{T}\left\langle\theta^{\varepsilon}(t, \cdot), y_{i, i}(t, \cdot)\right\rangle \mathrm{d} t=0 .
\end{aligned}
$$


Again, taking the limit $\epsilon \rightarrow 0$ and using Lemmas 2.5 and 2.6 we conclude that $(u, \theta, q)$ fulfill (2.5).

From Lemma 2.6, it is immediately clear that $(u, \theta, q)$ satisfy (2.2). Using Lemma 1.4 from [4] again, it follows from Lemma 2.5 that

$$
u^{\varepsilon} \longrightarrow u \text { in } C^{0}\left([0, T],\left(L^{2}\left(\Gamma_{C}\right)\right)^{n}\right) .
$$

Therefore, since

we conclude that

$$
\frac{1}{\varepsilon} \int_{\Gamma_{C}}\left|\left(u_{\nu}^{\varepsilon}(t, \cdot)-g(\cdot)\right)^{+}\right|^{2} \mathrm{~d} \Gamma \leq C,
$$

$$
\int_{\Gamma_{C}}\left|\left(u_{\nu}(t, \cdot)-g(\cdot)\right)^{+}\right|^{2} \mathrm{~d} \Gamma=0
$$

and therefore (2.6) is satisfied.

Note that we did not use $\mu>0$ yet. Therefore everything we proved so far will also hold if $\mu=0$. The critical part is in fact the convergence of quadratic terms that appear in (2.3), as we will see in the following calculations.

For any $w \in L^{\infty}\left(H_{\Gamma_{D}}^{1}\right) \cap W^{1, \infty}\left(L^{2}\right)$ we substitute $w_{p}$ in (2.16) by $w-u$ and obtain

$$
\begin{aligned}
& \left\langle\partial_{t}^{2} u^{\varepsilon}, w-u^{\varepsilon}\right\rangle+\left\langle C_{i j k l} u_{k, l}^{\varepsilon}, w_{i, j}-u_{i, j}^{\varepsilon}\right\rangle \\
& +\mu\left\langle\partial_{t} u_{i, j}^{\varepsilon}, w_{i, j}-u_{i, j}^{\varepsilon}\right\rangle+\left\langle m_{i j} \theta^{\varepsilon}, w_{i, j}-u_{i, j}^{\varepsilon}\right\rangle \\
= & -\frac{1}{\varepsilon} \int_{\Gamma_{C}}\left(u_{\nu}^{\varepsilon}-g\right)^{+}\left(w_{\nu}-u_{\nu}^{\varepsilon}\right) \mathrm{d} \Gamma-\varepsilon \int_{\Gamma_{C}} \partial_{t} u_{\nu}^{\varepsilon}\left(w_{\nu}-u_{\nu}^{\varepsilon}\right) \mathrm{d} \Gamma .
\end{aligned}
$$

Integrating from 0 to $T$ we arrive at

$$
\begin{aligned}
& \left\langle\partial_{t} u^{\varepsilon}(T, \cdot), w(T, \cdot)\right\rangle-\left\langle\partial_{t} u^{\varepsilon}(T, \cdot), u^{\varepsilon}(T, \cdot)\right\rangle-\left\langle u_{1}^{\varepsilon}, w(0, \cdot)-u_{0}^{\varepsilon}\right\rangle \\
& -\int_{0}^{T}\left\langle\partial_{t} u^{\varepsilon}(t, \cdot), \partial_{t} w(t, \cdot)\right\rangle \mathrm{d} t+\int_{0}^{T}\left\langle C_{i j k l} u_{k, l}^{\varepsilon}(t, \cdot), w_{i, j}(t, \cdot)\right\rangle \mathrm{d} t \\
+ & \int_{0}^{T}\left\langle\partial_{t} u^{\varepsilon}(t, \cdot), \partial_{t} u^{\varepsilon}(t, \cdot)\right\rangle \mathrm{d} t-\int_{0}^{T}\left\langle C_{i j k l} u_{k, l}^{\varepsilon}(t, \cdot), u_{i, j}^{\varepsilon}(t, \cdot)\right\rangle \mathrm{d} t \\
+ & \mu \int_{0}^{T}\left\langle\partial_{t} u_{i, j}^{\varepsilon}(t, \cdot), w_{i, j}(t, \cdot)\right\rangle \mathrm{d} t-\frac{\mu}{2}\left(\left\|\nabla u^{\varepsilon}(T, \cdot)\right\|^{2}-\left\|\nabla u_{0}^{\varepsilon}\right\|^{2}\right) \\
& +\int_{0}^{T}\left\langle m_{i j} \theta^{\varepsilon}(t, \cdot), w_{i, j}(t, \cdot)\right\rangle \mathrm{d} t-\int_{0}^{T}\left\langle m_{i j} \theta^{\varepsilon}(t, \cdot), u_{i, j}^{\varepsilon}(t, \cdot)\right\rangle \mathrm{d} t \\
= & \frac{1}{\varepsilon} \int_{0}^{T} \int_{\Gamma_{C}}\left(u_{\nu}^{\varepsilon}(t, \cdot)-g\right)^{+}\left(u_{\nu}^{\varepsilon}(t, \cdot)-g\right)-\left(u_{\nu}^{\varepsilon}(t, \cdot)-g\right)^{+}\left(w_{\nu}(t, \cdot)-g\right) \mathrm{d} \Gamma \mathrm{d} t \\
& -\varepsilon \int_{0}^{T} \int_{\Gamma_{C}} \partial_{t} u_{\nu}^{\varepsilon}(t, \cdot) w_{\nu}(t, \cdot) \mathrm{d} \Gamma \mathrm{d} t+\frac{\varepsilon}{2} \int_{\Gamma_{C}}\left|u_{\nu}^{\varepsilon}(T, \cdot)\right|^{2}-\left|u_{0 \nu}^{\varepsilon}\right|^{2} \mathrm{~d} \Gamma \\
\geq & -\varepsilon\left(\int_{0}^{T} \int_{\Gamma_{C}} \partial_{t} u_{\nu}^{\varepsilon}(t, \cdot) w_{\nu}(t, \cdot) \mathrm{d} \Gamma \mathrm{d} t+\frac{1}{2} \int_{\Gamma_{C}}\left|u_{\nu}^{\varepsilon}(T, \cdot)\right|^{2}-\left|u_{0 \nu}^{\varepsilon}\right|^{2} \mathrm{~d} \Gamma\right) .
\end{aligned}
$$

Using Lemmas 2.5 and 2.6, we see that the right-hand side of (2.38) will converge to 0 as $\epsilon \rightarrow 0$, since weak-* convergent series are bounded in norm. For the left-hand side we can again conclude the convergence of all terms that are linear in $\left(u^{\epsilon}, \theta^{\epsilon}, q^{\epsilon}\right)$. However, the convergence of the quadratic terms, namely the $L^{2}(0, T)$-norms of $\left\|u_{t}^{\epsilon}\right\|,\left\langle C_{i j k l} u_{i, j}, u_{k, l}\right\rangle$ 
and $\left\langle m_{i j} \theta, u_{i, j}\right\rangle$, remains an issue. While we know the terms will be bounded, we cannot conclude their convergence to the respective terms for $u$, as weak-* convergence does not imply norm convergence.

Note that it is not possible to circumvent this problem by simply taking estimates for the second order energy and giving a strong solution, since the second order energy is not (trivially) bounded in $\epsilon$. We remark that Muñoz Rivera and Racke [6] encountered a similar problem, which could be circumvented by reducing the problem to the radially symmetrical case and using an estimate obtained via compensated compactness. However, it is not possible to utilize this for our problem, since we do not have a bound on $\nabla \theta$, which is a necessary component of the proof in [6].

Therefore, we shall use $\mu>0$, which will yield

$$
u^{\varepsilon} \rightarrow u \text { in } W^{1,2}\left(\left(H_{\Gamma_{C}}^{1}\right)^{n}\right)
$$

by Lemma 2.5. From this we can conclude the uniform convergence of $u_{t}^{\epsilon}$ as well as $\nabla u$. It is then possible to take the limit $\epsilon \rightarrow 0$ in (2.38) and conclude that $(u, \theta, q)$ will satisfy (2.3).

3. Stability. In general, one cannot expect the exponential stability of a thermoelastic problem that is not radially symmetric. Therefore, we shall restrict our problem to the radially symmetric, isotropic and homogeneous cases; i.e., we assume that the following conditions hold:

The domain $\Omega$ is radially symmetric, in this case annular: $\Omega=B(0,1) \backslash B\left(0, r_{0}\right)$, $1>r_{0}>0 ; \quad \Gamma_{D}=\partial B\left(0, r_{0}\right) ; \quad \Gamma_{C}=\partial B(0,1) ; \quad \Gamma_{N}=\emptyset$.

The coefficients satisfy the following symmetry conditions:

$$
\begin{gathered}
C_{i j k l}=\lambda \delta_{i j} \delta_{k l}+\nu\left(\delta_{i k} \delta_{j l}+\delta_{j k} \delta_{i l}\right), \\
m_{i j}=\bar{m} \delta_{i j}, \quad K_{i j}=\kappa \delta_{i j}, \quad g(x)=\bar{g} \geq 0 \forall x \in \Gamma_{C} .
\end{gathered}
$$

Additionally, we shall assume that the solution to the problem as derived in the previous section is unique in this case, which implies that with radially symmetric initial data and the above assumptions on the coefficients, the solution itself will be radially symmetric. We shall first investigate the stability of the penalized problem, which will transfer to the original problem by a simple continuity argument.

With our assumptions, the equations take the form

$$
\begin{gathered}
\partial_{t}^{2} u^{\varepsilon}-\mu \partial_{t} \Delta u^{\varepsilon}-\lambda_{1} \Delta u^{\varepsilon}-\left(\lambda_{1}+\lambda_{2}\right) \nabla \operatorname{div} u^{\varepsilon}+\bar{m} \nabla \theta^{\varepsilon}=0, \\
\partial_{t} \theta^{\varepsilon}+\operatorname{div} q^{\varepsilon}+\bar{m} \operatorname{div} \partial_{t} u^{\varepsilon}=0 \\
\tau_{0} \partial_{t} q^{\varepsilon}+q^{\varepsilon}+\kappa \theta^{\varepsilon}=0
\end{gathered}
$$

with Lamé-Moduli $\lambda_{1}, \lambda_{2}$ satisfying $2 \lambda_{1}+n \lambda_{2}>0$ and constants $\kappa>0$ and $m \neq 0$.

The boundary conditions to the penalized problem then read

$$
\begin{gathered}
\left.\theta^{\varepsilon}\right|_{\partial \Omega}=0,\left.u^{\varepsilon}\right|_{\Gamma_{D}}=0, \\
\mu \partial_{t} \frac{\partial u^{\varepsilon}}{\partial \nu} \cdot \nu+\lambda_{1} \frac{\partial u^{\varepsilon}}{\partial \nu} \cdot \nu+\left(\lambda_{1}+\lambda_{2}\right) \operatorname{div} u^{\varepsilon}=-\frac{1}{\varepsilon}\left(u_{\nu}^{\varepsilon}-\bar{g}\right)^{+}-\varepsilon \partial_{t} u_{\nu}^{\varepsilon} \quad \text { on } \Gamma_{C} .
\end{gathered}
$$


As mentioned above, solutions to this problem will also be radially symmetric, so we can write

$$
u^{\varepsilon}(t, x)=x w(t,|x|), \theta^{\varepsilon}(t, x)=\psi(t,|x|), q^{\varepsilon}(t, x)=x h(t,|x|) .
$$

Writing $r:=|x|,(w, \psi, h)$ will then satisfy the equations

$$
\begin{gathered}
\partial_{t}^{2} w-\mu \partial_{t} w_{r} r-\mu \partial_{t} \frac{1}{r} w_{r}-\nu_{1} w_{r r}-\frac{\nu_{2}}{r} w_{r}+\frac{\bar{m}}{r} \theta_{r}=0, \\
\partial_{t} \psi+n h+r h_{r}+\bar{m} n \partial_{t} w+\bar{m} r \partial_{t} w_{r}=0 \\
\tau_{0} \partial_{t} h+h+\frac{\kappa}{r} \psi_{r}=0 .
\end{gathered}
$$

We will now show that the energy of the penalized problem, defined by

$$
E^{\varepsilon}(t):=\left\|\partial_{t} u^{\varepsilon}\right\|^{2}+\lambda_{1}\left\|\nabla u^{\varepsilon}\right\|^{2}+\kappa\left\|\theta^{\varepsilon}\right\|^{2}+\left\|q^{\varepsilon}\right\|^{2}+\frac{1}{\varepsilon} \int_{\Gamma_{C}}\left|\left(u_{\nu}^{\varepsilon}-\bar{g}\right)^{+}\right|^{2} \mathrm{~d} \Gamma,
$$

decays exponentially as time goes to infinity, i.e.

$$
E^{\varepsilon}(t) \leq \alpha E_{0}^{\varepsilon} e^{-\beta t} .
$$

We will use the technique of a Lyapunov functional, constructing the negative terms of the energy and combining the respective functionals in a final estimate. First, one easily sees by multiplying (3.1) with $\partial_{t} u$, (3.2) with $\kappa \theta$ and (3.3) with $q$ and integrating over $\Omega$, that the energy satisfies

$$
\frac{\mathrm{d}}{\mathrm{d} t} E^{\varepsilon}(t) \leq-C_{2}\left(\mu\left\|\partial_{t} u^{\varepsilon}\right\|_{n}^{2}+\left\|q^{\varepsilon}\right\|_{n}^{2}\right) .
$$

Lemma 3.1. Let

$$
F_{1}(t):=\left\langle\partial_{t} u^{\varepsilon}, u^{\varepsilon}\right\rangle_{n}+\varepsilon \int_{\Gamma_{C}}\left|u_{\nu}^{\varepsilon}\right|^{2} \mathrm{~d} \Gamma-\mu|| \nabla u^{\varepsilon} \|_{n \times n}^{2} .
$$

Then for any $\delta_{1}>0$,

$$
\frac{\mathrm{d}}{\mathrm{d} t} F_{1}(t) \leq-\left(C_{3}-\delta_{1}\right)\left\|\nabla u^{\varepsilon}\right\|_{n \times n}^{2}-\frac{1}{\varepsilon} \int_{\Gamma_{C}}\left|\left(u_{\nu}^{\varepsilon}-\bar{g}\right)^{+}\right|^{2} \mathrm{~d} \Gamma+\left\|\partial_{t} u^{\varepsilon}\right\|_{n}^{2}+\frac{C_{4}}{\delta_{1}}\left\|\theta^{\varepsilon}\right\|^{2} .
$$

Proof.

$$
\begin{aligned}
\frac{\mathrm{d}}{\mathrm{d} t}\left\langle\partial_{t} u^{\varepsilon}, u^{\varepsilon}\right\rangle= & \left\|\partial_{t} u^{\varepsilon}\right\|^{2}+\left\langle\partial_{t}^{2} u^{\varepsilon}, u^{\varepsilon}\right\rangle \\
= & \left\|\partial_{t} u^{\varepsilon}\right\|^{2}-\lambda_{1}\left\|\nabla u^{\varepsilon}\right\|^{2}-\mu\left\langle\partial_{t} \nabla u^{\varepsilon}, \nabla u^{\varepsilon}\right\rangle-\left\langle\bar{m} \theta^{\varepsilon}, \operatorname{div} u^{\varepsilon}\right\rangle \\
& -\left(\lambda_{1}+\lambda_{2}\right)\left\|\operatorname{div} u^{\varepsilon}\right\|^{2}-\frac{1}{\varepsilon} \int_{\Gamma_{C}}\left(u_{\nu}^{\varepsilon}-\bar{g}\right)^{+} u_{\nu} \mathrm{d} \Gamma-\varepsilon \int_{\Gamma_{C}} \partial_{t} u_{\nu}^{\varepsilon} u_{\nu}^{\varepsilon} \mathrm{d} \Gamma .
\end{aligned}
$$

Estimating

$$
\left|\left\langle\bar{m} \theta^{\varepsilon}, \operatorname{div} u^{\varepsilon}\right\rangle\right| \leq C_{4}\left(\delta_{1}\left\|\nabla u^{\varepsilon}\right\|^{2}+\frac{1}{\delta},\left\|\theta^{\varepsilon}\right\|^{2}\right)
$$

for any $\delta_{1}>0$ and

$$
-\frac{1}{\varepsilon} \int_{\Gamma_{C}}\left(u_{\nu}^{\varepsilon}-\bar{g}\right)^{+} u_{\nu}^{\varepsilon} \mathrm{d} \Gamma \leq-\frac{1}{\varepsilon} \int_{\Gamma_{C}}\left|\left(u_{\nu}^{\varepsilon}-\bar{g}\right)^{+}\right|^{2} \mathrm{~d} \Gamma,
$$

we obtain the desired result. 
LEMma 3.2. Let

$$
\Psi(t, r):=\int_{r_{0}}^{r} \psi(t, s) \mathrm{d} s
$$

and

$$
F_{2}(t):=-\tau_{0} \int_{r_{0}}^{1} \Psi(t, r) h(t, r) \mathrm{d} r
$$

Then for any $\delta_{2}, \delta_{3}>0$,

$$
\frac{\mathrm{d}}{\mathrm{d} t} F_{2}(t) \leq \frac{C}{\delta_{2}+\delta_{3}} \int_{r_{0}}^{1}|h(t, r)|^{2} \mathrm{~d} r-\frac{\kappa-\delta_{2}}{r_{0}} \int_{r_{0}}^{1}|\psi(t, r)|^{2} \mathrm{~d} r+\delta_{3} \int_{r_{0}}^{1}\left|\partial_{t} w(t, r)\right|^{2} \mathrm{~d} r .
$$

Proof. By (3.6), $\Psi$ satisfies

$$
\partial_{t} \Psi+\int_{r_{0}}^{r} n h \mathrm{~d} s+\int_{r_{0}}^{r} s h \mathrm{~d} s+\int_{r_{0}}^{r} \bar{m} n \partial_{t} w \mathrm{~d} s+\int_{r_{0}}^{r} \bar{m} s \partial_{t} w_{s} \mathrm{~d} s=0 .
$$

Multiplying (3.12) with $h r$ and integrating, we obtain for any $\delta_{3}>0$,

$$
\begin{aligned}
-\int_{r_{0}}^{1}\left(\partial_{t} \Psi\right) h r \mathrm{~d} r & =n \int_{r_{0}}^{1} h r \int_{r_{0}}^{r} h \mathrm{~d} s \mathrm{~d} r+\int_{r_{0}}^{1} h r \int_{r_{0}}^{r} s h_{s} \mathrm{~d} s \mathrm{~d} r \\
& +\bar{m} n \int_{r_{0}}^{1} h r \int_{r_{0}}^{r} \partial_{t} w(t, s) \mathrm{d} s \mathrm{~d} r+\bar{m} \int_{r_{0}}^{1} h \int_{r_{0}}^{r} s \partial_{t} w_{s} \mathrm{~d} s \mathrm{~d} r \\
& \leq \frac{C}{\delta_{3}} \int_{r_{0}}^{1}|h(t, r)|^{2} \mathrm{~d} r+\delta_{3} \int_{r_{0}}^{1}|w(t, r)|^{2} \mathrm{~d} r .
\end{aligned}
$$

Multiplying (3.7) by $\Psi r$ and integrating, we obtain

$$
\tau_{0} \int_{r_{0}}^{1} \Psi\left(\partial_{t} h\right) r \mathrm{~d} r+\int_{r_{0}}^{1} \Psi h r \mathrm{~d} r+\int_{r_{0}}^{1} \kappa \Psi_{r r} \Psi \mathrm{d} r=0
$$

We have, by definition of $\Psi$,

$$
\int_{r_{0}}^{1} \kappa \Psi_{r r}(t, r) \Psi(t, r) \mathrm{d} r=-\kappa \int_{r_{0}}^{1}\left|\Psi_{r}(t, r)\right|^{2} \mathrm{~d} r=-\kappa \int_{r_{0}}^{1}|\psi(t, r)|^{2} \mathrm{~d} r .
$$

Using Poincaré's Inequalitiy for $\Psi$, this implies for any $\delta_{2}>0$,

$$
-\int_{r_{0}}^{1} \Psi\left(\partial_{t} h\right) r \mathrm{~d} r \leq \frac{C}{\delta_{2}} \int_{r_{0}}^{1}|h|^{2} \mathrm{~d} r-\left(\kappa-\delta_{2}\right) \int_{r_{0}}^{1}|\psi|^{2} \mathrm{~d} r .
$$

Combining (3.13) and (3.14), we obtain the desired result.

Defining

$$
L(t):=N E^{\varepsilon}(t)+F_{1}(t)+\delta_{4} F_{2}(t),
$$

where $\delta_{4}$ will be chosen later, we easily see that for large enough $N$ there exist $C_{1}, C_{2}>0$ such that

$$
C_{1} E(t) \leq L(t) \leq C_{2} E(t) .
$$

Now, we can prove the essential theorem of this section.

Theorem 3.3. Let $\mu>0$. Then the system is exponentially stable; i.e., there is a $\beta>0$ such that

$$
E^{\varepsilon}(t) \leq \alpha E_{0}^{\varepsilon} e^{-\beta t}
$$


Proof. Using (3.8), (3.9) and (3.11), we conclude that

$$
\begin{aligned}
\frac{\mathrm{d}}{\mathrm{d} t} L(t) \leq & -N C_{2}\left(\mu\left\|\partial_{t} u^{\varepsilon}\right\|_{n}^{2}+\left\|q^{\varepsilon}\right\|_{n}^{2}\right)-\left(C_{3}-\delta_{1}\right)\left\|\nabla u^{\varepsilon}\right\|_{n \times n}^{2} \\
& -\frac{1}{\varepsilon} \int_{\Gamma_{C}}\left|\left(u_{\nu}^{\varepsilon}-\bar{g}\right)^{+}\right|^{2} \mathrm{~d} \Gamma+\left\|\partial_{t} u^{\varepsilon}\right\|_{n}^{2}+\frac{C_{4}}{\delta_{1}} \|\left.\theta^{\varepsilon}\right|^{2} \\
& +\delta_{4}\left(\frac{C_{5}}{\delta_{2}+\delta_{3}} \int_{r_{0}}^{1}|h(t, r)|^{2} \mathrm{~d} r-\frac{\kappa-\delta_{2}}{r_{0}} \int_{r_{0}}^{1}|\psi|^{2} \mathrm{~d} r+\delta_{3} \int_{r_{0}}^{1}\left|\partial_{t} w\right|^{2} \mathrm{~d} r\right) \\
\leq & \left(1+C_{6} \delta_{3} \delta_{4}-N C_{2} \mu\right)\left\|\partial_{t} u^{\varepsilon}\right\|_{n}^{2}+\left(\frac{\delta_{4} C_{7}}{\delta_{2}+\delta_{3}}-N C_{2}\right)\left\|q^{\varepsilon}\right\|_{n}^{2}+\left(\delta_{1}-C_{3}\right)\left\|\nabla u^{\varepsilon}\right\|_{n \times n}^{2} \\
& -\frac{1}{\varepsilon} \int_{\Gamma_{C}}\left|\left(u_{\nu}^{\varepsilon}-\bar{g}\right)^{+}\right|^{2} \mathrm{~d} \Gamma+\left(\frac{C_{4}}{\delta_{1}}-\delta_{4} \frac{\kappa-\delta_{2}}{r_{0}}\right)\left\|\theta^{\varepsilon}\right\|^{2} .
\end{aligned}
$$

Choosing $\delta_{1}<C_{3}$ and $\delta_{2}<\kappa$, then $\delta_{4}>\frac{r_{0} C_{4}}{\delta_{1}\left(\kappa-\delta_{2}\right)}$ and (arbitrarily) $\delta_{3}=1$, we conclude that, for sufficiently large $N$, there is a $C>0$ such that

$$
\frac{\mathrm{d}}{\mathrm{d} t} L(t) \leq-C E^{\varepsilon}(t)
$$

Using (3.15), this proves our theorem.

Note that $\delta_{3}$ is not really needed for the construction of the Lyapunov functional and could have been left as 1 . However, we want to point out that the positive $u_{t}$ term arising from $F_{2}$ is not a problem; the problem requiring $\mu>0$ is the positive $u_{t}$ term arising from $F_{1}$, which cannot be made arbitrarily small without losing the negative terms for the derivatives of $u$. For the classical problem, Muñoz Rivera and Racke [6] showed that this term can be handled by adding additional functions to the Lyapunov functional; however, this gives rise to a positive $\nabla \theta$ term. While $\nabla \theta$ is given as a negative term from the energy itself in the classical case, this does not hold for $\tau_{0}>0$; in fact we do not know anything about derivatives of $\theta$. It is therefore necessary to gain the negative $u_{t}$ term by other means, one of them being the viscoelastic term. If we define the energy of the original problem as

$$
E(t):=\left\|\partial_{t} u\right\|^{2}+\|\nabla u\|^{2}+\|\theta\|^{2}+\|q\|^{2},
$$

we see by the lower semicontinuity of the norms of weak*-convergent series, using Lemma 2.5, that

$$
\liminf _{\varepsilon \rightarrow 0} E^{\varepsilon}(t) \geq E(t) .
$$

Using the strong convergence of initial data, we obtain

$$
E(t) \leq E^{\varepsilon}(t) \leq \alpha E^{\varepsilon}(0) \exp (-\beta t) \rightarrow \alpha E(0) \exp (-\beta t) .
$$

This proves our final theorem:

Theorem 3.4. Let $\mu>0$. Then there are $\alpha, \beta>0$ such that

$$
E(t) \leq \alpha E(0) \exp (-\beta t)
$$




\section{REFERENCES}

[1] K. A. Ames and L. E. Payne, Uniqueness and continuous dependence of solutions to a multidimensional thermoelastic contact problem, J. Elasticity 34 (1994), 139-148. MR.1288855 (95f:73004)

[2] C. M. Elliott and Q. Tang, A dynamic contact problem in thermoelasticity, Nonlinear Analysis 23 (1994), 883-898. MR 1302150 (95i:73013)

[3] H. Gao and J. Muñoz Rivera, Global existence and decay for the semilinear thermoelastic contact problem, J. Differential Equations 186 (2002), 52-68. MR.1941092(2004a:74010)

[4] J.U. Kim, A boundary thin obstacle problem for a wave equation, Comm. Partial Differential Equations 14 (1989), 1011-1026. MR.1017060 (91a:35121)

[5] J. Muñoz Rivera and S. Jiang, The thermoelastic and viscoelastic contact of two rods, J. Math. Anal. Appl. 217 (1998), 423-458. MR1492098 (98m:73076)

[6] J. Muñoz Rivera and R. Racke, Multidimensional contact problems in thermoelasticity, SIAM J. Appl. Math. 58 (1998), 1307-1337. MR.1628692 (99f:73015)

[7] H. Ferñandez Sare and R. Racke, On the stability of damped Timoshenko systems - Cattaneo versus Fourier law, Archive Rational Mech. Anal. (to appear) (2007).

[8] P. Shi and M. Shillor, Existence of a solution to the $N$-dimensional problem of thermoelastic contact, Comm. Partial Differential Equations 17 (1992), 1597-1618. MR1187623 (93i:73052) 